\title{
Comparison of the Magnetic Resonance Imaging Findings of Paget's Disease of the Breast and Malignant Tumor Invasion of the Nipple-Areola Complex
}

\author{
(1) Almila Coşkun Bilge, (1) Hale Aydın, (1) Işıl Esen Bostancı, (1) Özge Tanişman, (D) Diba Saygıılı Öz \\ Department of Radiology, Dr Abdurrahman Yurtaslan Ankara Oncology Training and Research Hospital, Ankara, Turkey
}

\begin{abstract}
Objective: We aimed to investigate the distinction between Paget's disease of the breast (PDB) and malignant tumor invasion of nipple-areolar complex (MTION) with Magnetic resonance imaging (MRI) findings without the need for skin punch biopsy.

Materials and Methods: MRI findings of 16 patients with pathologically proven PDB and 11 patients with pathologically proven MTION were reviewed retrospectively. MRI images were assessed for nipple morphological changes; areolar-periareolar skin changes; thickness, classification, and kinetic characteristics of the nipple-areolar complex (NAC) enhancement; morphological pattern, size, and pathological diagnosis of concomitant malignant lesions; kinetic characteristics of the concomitant malignant lesions enhancement; continuity of enhancement between the nipple and closest concomitant malignant lesion; similarity of enhancement kinetics of the NAC and concomitant malignant lesions; and nipple-to-malignant lesion distance in both patient groups.

Results: Areolar-periareolar skin thickening was statistically different between the patient groups. Enhancement kinetic pattern was classified as persistent in four patients with MTION and plateau in seven patients with PDB. Moreover, NAC enhancement kinetic characteristics were statistically different between the groups. Invasive ductal carcinoma was detected in three patients with PDB and five patients with MTION. A statistically significant difference in malignant lesion pathological types was detected between the patient groups.

Conclusion: The significant MRI findings in patients with MTION diagnosed as invasive ductal carcinoma were areolar-periareolar skin thickening and asymmetric NAC enhancement with persistent kinetics pattern. In patients diagnosed with ductal carcinoma in situ, a plateau pattern of asymmetric NAC enhancement without any areolar-periareolar skin changes on MRI may indicate PDB.
\end{abstract}

Keywords: MRI, breast imaging, nipple-areola complex, breast cancer

Cite this article as: Coşkun Bilge A, Aydın H, Esen Bostancı I, Tanişman Ö, Saygılı Öz D. Comparison of the Magnetic Resonance Imaging Findings of Paget's Disease of the Breast and Malignant Tumor Invasion of the Nipple-Areola Complex. Eur J Breast Health 2021; 17(3): 265-273

\section{Key Points}

- The nipple-areola complex may be affected by malignancies.

- Dynamic breast MRI findings help us to diagnose Paget's disease of the breast and malignant tumor invasion of the nipple-areolar complex without skin punch biopsy.

- Areolar-periareolar skin changes and the kinetic pattern of nipple-areola complex enhancement and pathologic diagnosis of the concomitant malignant lesion are critical MRI findings in the differentiation of Paget's disease of the breast and malignant tumor invasion of the nipple-areolar complex.

- $\quad$ 3.0-T MRI is superior to 1.5-T MRI in obtaining areolar-periareolar skin thickness.

\section{Introduction}

The nipple-areola complex (NAC) is a specialized breast structure for breastfeeding that involves the pigmented squamous epithelium, a layer of circumferential smooth muscle, and sebaceous glands (1). The skin of the nipple is continuous with the epithelium of the ducts (2). Cancer of the ducts may spread to the NAC (3).

The NAC may be affected by malignancies such as invasive cancer, ductal carcinoma in situ (DCIS), or Paget's disease of the breast (PDB) (4). NAC involvement in breast cancers has an incidence rate of 5.6\%-24.6\% (5). The different pathological mechanisms of NAC involvement include a direct tumor invasion and dissemination of the tumor within ducts or lymphatics (6). Tumor size and distance between the tumor 
and nipple are significant factors for NAC involvement. Tumors that are small and far from the nipple pose lower risk of NAC involvement $(5,7)$. PDB is a rare malignant entity that accounts for $0.7 \%-4.9 \%$ of all breast carcinomas. PDB is usually accompanied by underlying invasive cancer or DCIS (8). Two hypotheses regarding the etiopathology of PDB were noted. The epidermotropic theory suggests that ductal cancer cells migrate through the basement membrane, whereas another theory implicates malignant neoplastic transformation of the intraepidermal clear cells of the NAC (9, 10 ), which may explain the absence of an underlying malignancy in $1 \%-6 \%$ of PDB cases (8). Definitive diagnosis of diseases of the NAC is made by histopathological examination of wedge biopsy and mastectomy or lumpectomy samples (9). The NAC is examined using imaging methods before skin punch biopsy. Magnetic resonance imaging (MRI) has a higher sensitivity than mammography and ultrasonography in evaluating diseases of the NAC and underlying malignancy $(5,11)$.

There are no previous studies in the literature comparing the MRI findings of PDB and malignant tumor invasion of the NAC (MTION). Therefore, this study aimed to differentiate between PDB and MTION by dynamic breast MRI findings without the need for skin punch biopsy.

\section{Materials and Methods}

This study was approved by the Medical Ethics Committee of Dr Abdurrahman Yurtaslan Ankara Oncology Training and Research Hospital and was in accordance with the principles of the Declaration of Helsinki (approval number: 2020-10/834; approval date: October $\left.21^{\text {st }}, 2020\right)$.

\section{Study population}

We reviewed the pathology results of patients who underwent preoperative dynamic breast MRI between June 2016 and September 2019. Patients who had preoperative breast MRI examination images in our archive and were diagnosed as having PDB or MTION as a result of pathology examination of operative material were included in this study. Patients who received neoadjuvant chemotherapy or did not have postoperative pathology report of PDB or MTION were excluded.

Of 620 patients who underwent preoperative breast MRI examination, 35 patients were diagnosed by pathologic examination as having nipple malignancy such as PDB or MTION. Eight patients received neoadjuvant chemotherapy before surgery and were excluded. Finally, the study group included 27 patients with a mean age of 51.4 years (age range: 31-76 years). All patients were women and underwent mastectomy within 1 month of breast MRI. Pathology reports indicated PDB in 16 patients and MTION in 11 patients.

\section{MRI technique}

Among the patients, 17 were examined with a 1.5 -Tesla $(\mathrm{T})$ magnetic resonance (MR) scanner (Signa HDx; GE Healthcare, Wisconsin, USA) and 10 with a 3.0-T MR scanner (Skyra; Siemens Healthineers, Erlangen, Germany) before surgery. All patients were examined in the prone position using a breast array coil. Imaging parameters on the 1.5-T MR scanner were as follows: TR/TE, 6500/45; TI, $150 \mathrm{~ms}$; field of view (FOV), $320 \mathrm{~mm}$; matrix, $416 \times$ 224; and number of excitations (NEX), 1 and slice thickness (ST) $\mathrm{mm}$; matrix, $448 \times 224$; and NEX, 1 and ST of $5 \mathrm{~mm}$ for axial T1weighted (T1W) images; TR/TE, 4/1.5; FA, 10 ; FOV, $320 \mathrm{~mm}$; matrix, $350 \times 350$; and NEX, 1 and ST of $2.8 \mathrm{~mm}$ for dynamic axial fat-suppressed T1W images (before and after contrast injection); and TR/TE, 1000/83; FOV, 320 mm; matrix, $192 \times 192$; and NEX, 4 and ST of $5 \mathrm{~mm}$ for echo-planar imaging (EPI)-based diffusion weighted imaging (DWI). In contrast, the imaging parameters on 3.0-T MR scanner were as follows: TR/TE, 5000/88; FOV, $350 \mathrm{~mm}$; matrix, $576 \times 768$; NEX, 2; and ST, $4 \mathrm{~mm}$ for axial fat-saturated T2-weighted (T2W) sequences; TR/TE, 800/11; FOV, 350 mm; matrix, $448 \times 640$; NEX, 1; and ST, $4 \mathrm{~mm}$ for axial T1W images; TR/TE, 4.3/1.6; FA, 10 FOV, 350 mm; matrix, $352 \times 352$; NEX, 1 ; and ST, $1 \mathrm{~mm}$ for dynamic axial gradient fat-suppressed T1W images (before and after contrast injection); and TR/TE, 6000/85; FOV, $361 \times 401 \mathrm{~mm}$; matrix, $180 \times 200$; NEX, 1; and ST, $5 \mathrm{~mm}$ for EPI-based DWI. Gadobutrol/gadopentetate dimeglumine at of $0.1 \mathrm{mmol} / \mathrm{kg}$ was used as the contrast agent for dynamic contrastenhanced sequences. Images were taken once before contrast and five times after contrast injection. Standard subtraction images were generated by subtracting precontrast images from postcontrast images. Maximum-intensity projection (MIP) and multiplanar reconstruction images were reconstructed. Conventional kinetic analysis was done using a time-intensity curve for the NAC, mass lesions, and nonmass enhancing lesions.

\section{Analysis of magnetic resonance images}

MR images were evaluated retrospectively on two workstations by two radiologists who had at least 5 years of experience in breast imaging. The radiologists were blinded to the pathology reports. Each case was evaluated through a consensus between the two radiologists.

We evaluated nipple changes such as nipple inversion or retraction and skin thickness of the NAC on T1W and fat-suppressed T2W images. Complete and partial pulling inward of normal nipple was accepted as inversion and retraction of the nipple, respectively $(12,13)$. The thickness of the areolar-periareolar skin is normally between 0.5 and $2 \mathrm{~mm}$ (2). Asymmetric thickening of the areolar-periareolar skin relative to the contralateral breast was evaluated. The characteristic enhancement of the NAC is symmetric, with superficial linear enhancement thickness of 1-2 mm $(1,13)$. We evaluated thickness and classification of NAC enhancement on postcontrast T1W MR images. Symmetric absent, mild, and intense nipple enhancement patterns were considered normal (1). Asymmetric NAC enhancement was considered pathologic enhancement. Pathologic NAC enhancement was classified as nodular, discoid, linear, and irregular (11). We evaluated NAC enhancement on postcontrast T1W subtraction and MIP images. Concomitant malignant lesions were classified as single mass, multiple masses, nonmass enhancement (NME), or mass with NME. The largest dimension of the malignant lesions was recorded. Enhancement kinetics of the NAC and concomitant malignant lesions were evaluated as washout, plateau, or persistent enhancement. The enhancement patterns of the NAC and concomitant malignant lesions were compared. The continuity of contrast enhancement in the area between the nipple and subareolar mass or NME was evaluated and classified as discontinuous if a non-enhancing area was observed between the NAC and mass or NME. The distance between the nipple and malignant lesion was measured. If a gap between the mass or NME and NAC was not noted, it was classified as a continuous enhancement (12). The nipple-to-malignant lesion distance was accepted as $0 \mathrm{~mm}$ in these cases. 


\section{Statistical analysis}

SPSS 15.0 for Windows program was used for statistical analysis. As descriptive statistics, numbers and percentages are presented for categorical variables and mean, standard deviation, minimum, and maximum values for numerical variables. Student's t-test was used for between-group comparisons of normally distributed variables and Mann-Whitney $U$ test for non-normally distributed variables. Categorical variables were compared using chi-square test. Statistical significance was accepted at $\mathrm{p}<0.05$.

\section{Results}

Pathological evaluation determined 16 patients with PDB and 11 patients with MTION. MRI showed nipple inversion or retraction in 19 cases: nine patients $(56.3 \%)$ in the PDB group and 10 patients (90.9\%) in the MTION group. Nipple inversion was more common in patients with MTION (63.6\%) than in those with PDB (25\%); however, the difference was not significant $(\mathrm{p}=0.093)$ (Figure 1).

Areolar-periareolar skin thickening was detected in a total of 12 patients: two (12.5\%) in the PDB group and $10(90.9 \%)$ in the MTION group $(\mathrm{p}<0.001)$ (Figure 1$)$.

NAC enhancement was observed in 26 patients. In seven of these patients, enhancement was symmetrical, superficial, and linear, which

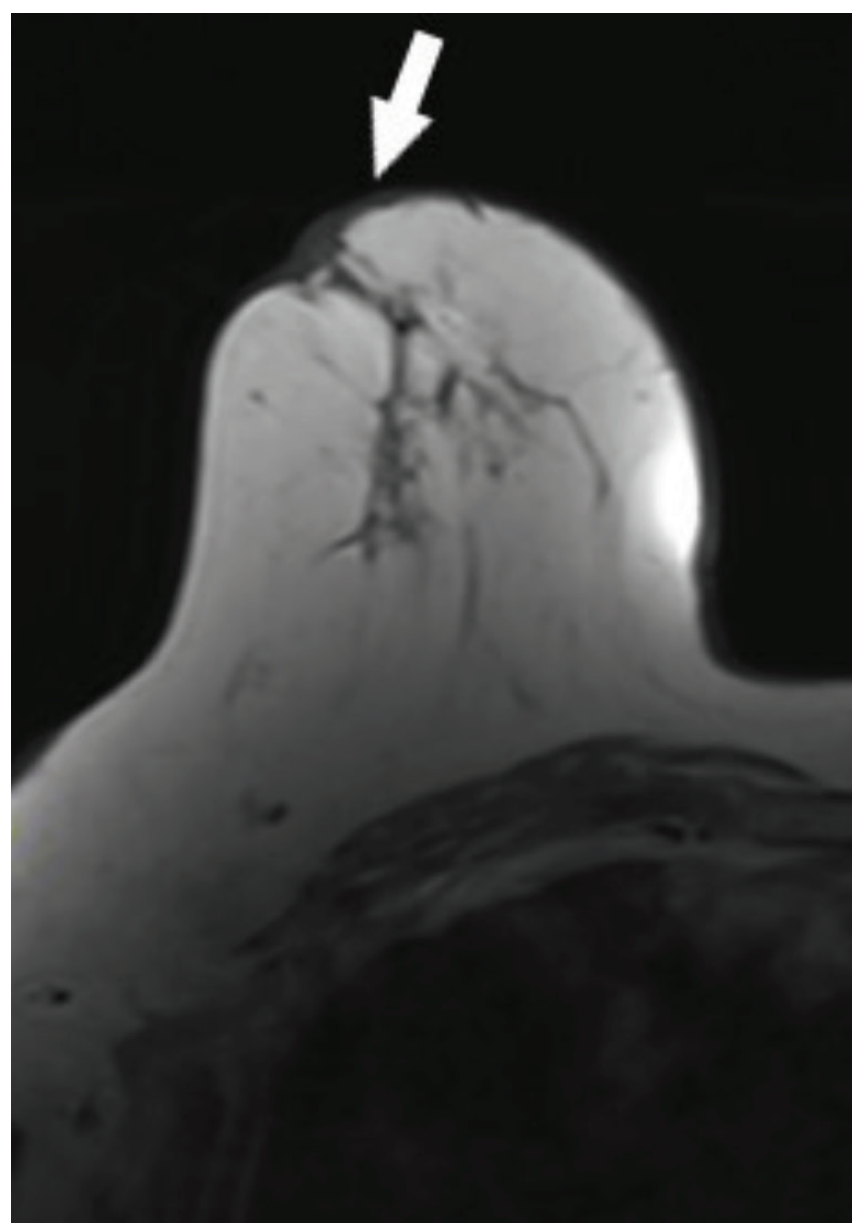

Figure 1. Axial T1-weighted MRI of a 66-year-old woman with ductal carcinoma in situ and malignant tumor invasion of the nipple-areola complex shows areolar-periareolar skin thickening (white arrow)

MRI: Magnetic resonance imaging was considered normal (Figure 2). Only one patient with PDB showed no NAC enhancement. Pathological NAC enhancement was detected in $12(75.1 \%)$ of the patients with PDB and seven $(63.7 \%)$ of the patients with MTION. Pathological NAC enhancement was evaluated as nodular in four, discoid in three, linear in two, and irregular in 10 patients. Irregular NAC enhancement was more common in both patient groups (Figure 3a). Enhancement patterns were not statistically different between the groups $(\mathrm{p}=0.086)$.

Median thickness of the NAC enhancement was $0.47 \pm 0.29 \mathrm{~cm}$ in the $\mathrm{PDB}$ group and $0.32 \pm 0.15 \mathrm{~cm}$ in the MTION group $(\mathrm{p}=0.141)$.

NAC enhancement kinetics are summarized in Table 1. Persistent enhancement pattern was more common in MTION and plateau enhancement pattern in PDB. The distribution of NAC enhancement kinetics differed significantly between the groups $(\mathrm{p}=0.026)$.

Morphological pattern of the concomitant malignant lesion was classified as mass in three, NME in 17, and mass with NME in seven patients. NME was the most common in both patient groups. The distribution of morphological patterns did not differ significantly between the groups $(\mathrm{p}=0.078)$. The median size of the concomitant malignant lesions was $2.99 \pm 2.12 \mathrm{~cm}$ in patients with $\mathrm{PDB}$ and $2.9 \pm 1.63 \mathrm{~cm}$ in patients with MTION ( $\mathrm{p}=0.863)$.

Concomitant malignant lesion enhancement kinetics were evaluated as washout, plateau, and persistent enhancement pattern in six (37.5\%), seven $(43.8 \%)$, and three $(18.8 \%)$ patients in the PDB group and four $(36.4 \%)$, three $(27.3 \%)$, and four $(36.4 \%)$ patients in the MTION group, respectively $(\mathrm{p}=0.551)$

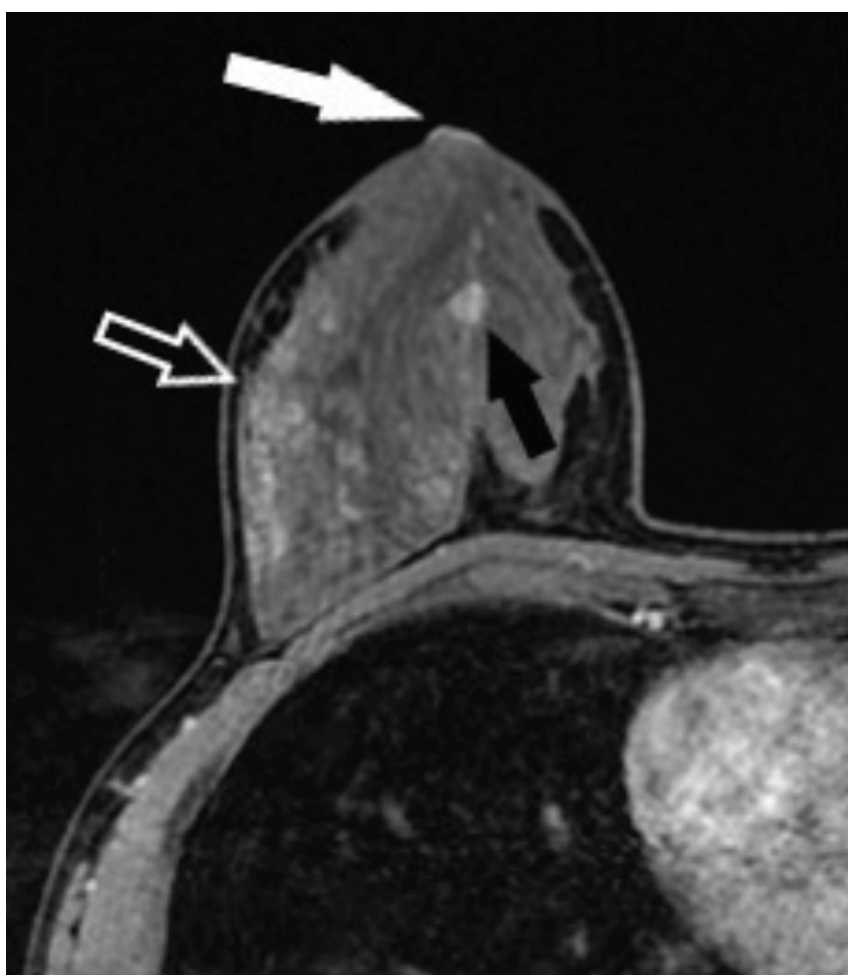

Figure 2. Axial dynamic postcontrast T1-weighted MRI of the right breast of a 45-year-old woman with invasive ductal carcinoma and Paget's disease of the breast shows a normal thin, superficial enhancement (white arrow) of the skin of the nipple-areola complex, concomitant mass (black arrow), and concomitant nonmass enhancement (empty white arrow)

MRI: Magnetic resonance imaging 
On comparison of the enhancement kinetic curves of the NAC and concomitant malignant lesions, different patterns were observed in nine patients $(56.3 \%)$ in the PDB group and eight patients $(72.7 \%)$ in the MTION group (Table 2; Figures $3 \mathrm{~b}$ and $3 \mathrm{c}$ ). There was no statistically significant difference between the groups $(\mathrm{p}=0.448)$.
The mean nipple-to-malignant lesion distances in the PDB and MTION groups were $0.29 \pm 0.64 \mathrm{~cm}$ and $0.06 \pm 0.16 \mathrm{~cm}$, respectively. The average distance was longer in patients with PDB; however, the difference was not statistically significant $(\mathrm{p}=0.799)$.

Table 1. MRI findings compared by pathologic diagnosis of nipple-areola complex involvement

\begin{tabular}{|c|c|c|c|}
\hline \multirow[b]{2}{*}{ MRI findings (n) } & \multicolumn{3}{|c|}{ Pathologic diagnosis of NAC involvement } \\
\hline & $\begin{array}{c}\text { PDB } \\
(n=16)(\%)\end{array}$ & $\begin{array}{c}\text { MTION } \\
(n=11)(\%)\end{array}$ & p-value \\
\hline \multicolumn{4}{|l|}{ Nipple change } \\
\hline None $(n=8)$ & $7(43.7 \%)$ & $1(9.1 \%)$ & \multirow{3}{*}{0.093} \\
\hline Inversion ( $n=11)$ & $4(25.0 \%)$ & $7(63.6 \%)$ & \\
\hline Retraction $(n=8)$ & $5(31.3 \%)$ & $3(27.3 \%)$ & \\
\hline \multicolumn{4}{|l|}{ Areolar/periareolar skin thickening } \\
\hline Absent $(n=15)$ & $14(87.5 \%)$ & $1(9.1 \%)$ & \multirow{2}{*}{$<0.001$} \\
\hline Present $(n=12)$ & $2(12.5 \%)$ & 10 (90.9\%) & \\
\hline \multicolumn{4}{|l|}{ NAC enhancement } \\
\hline \multicolumn{4}{|l|}{ Classification } \\
\hline None $(n=1)$ & $1(6.3 \%)$ & $0(0.0 \%)$ & \multirow{6}{*}{0.086} \\
\hline Normal $(n=7)$ & $3(18.7 \%)$ & $4(36.4 \%)$ & \\
\hline Nodular $(n=4)$ & $4(25.0 \%)$ & $0(0.0 \%)$ & \\
\hline Discoid $(n=3)$ & $3(18.7 \%)$ & $0(0.0 \%)$ & \\
\hline Linear $(n=2)$ & $0(0.0 \%)$ & $2(18.2 \%)$ & \\
\hline Irregular $(n=10)$ & $5(31.3 \%)$ & $5(45.4 \%)$ & \\
\hline Thickness (mm), mean \pm SD & $4.71 \pm 2.97$ & $3.22 \pm 1.55$ & 0.141 \\
\hline \multicolumn{4}{|l|}{ Kinetic pattern } \\
\hline Persistent ( $n=13$ ) & $4(26.7 \%)$ & $9(81.8 \%)$ & \multirow{3}{*}{0.026} \\
\hline Plateau $(n=8)$ & $7(46.6 \%)$ & $1(9.1 \%)$ & \\
\hline Washout $(n=5)$ & $4(26.7 \%)$ & $1(9.1 \%)$ & \\
\hline \multicolumn{4}{|l|}{ Concomitant malignant lesion } \\
\hline \multicolumn{4}{|l|}{ Morphological pattern } \\
\hline Single mass $(n=3)$ & $0(0.0 \%)$ & $3(27.3 \%)$ & \multirow{3}{*}{0.078} \\
\hline NME $(n=17)$ & $12(75.0 \%)$ & $5(45.4 \%)$ & \\
\hline NME + mass $(n=7)$ & $4(25.0 \%)$ & $3(27.3 \%)$ & \\
\hline Maximum size $(\mathrm{mm})$, mean \pm SD & $29.9 \pm 21.2$ & $29.0 \pm 16.3$ & 0.863 \\
\hline \multicolumn{4}{|l|}{ Enhancement kinetic pattern } \\
\hline Persistent $(n=7)$ & $3(18.7 \%)$ & $4(36.4 \%)$ & \multirow{3}{*}{0.551} \\
\hline Plateau $(n=10)$ & $7(43.8 \%)$ & $3(27.2 \%)$ & \\
\hline Washout $(n=10)$ & $6(37.5 \%)$ & $4(36.4 \%)$ & \\
\hline \multicolumn{4}{|l|}{ Pathological diagnosis } \\
\hline Inflammatory carcinoma $(n=2)$ & $2(12.5 \%)$ & $0(0.0 \%)$ & \multirow{5}{*}{0.025} \\
\hline Invasive ductal carcinoma $(n=8)$ & $3(18.7 \%)$ & $5(45.5 \%)$ & \\
\hline DCIS $(n=10)$ & $9(56.3 \%)$ & $1(9.1 \%)$ & \\
\hline Invasive breast carcinoma $(n=6)$ & $2(12.5 \%)$ & $4(36.3 \%)$ & \\
\hline Invasive lobular carcinoma $(n=1)$ & $0(0.0 \%)$ & $1(9.1 \%)$ & \\
\hline
\end{tabular}

NAC: Nipple-areola complex; MRI: Magnetic resonance imaging; PDB: Paget's disease of the breast; MTION: Malignant tumor invasion of the nipple-areola complex; SD: Standard deviation; NME: Nonmass enhancement; DCIS: Ductal carcinoma in situ; n: Number 


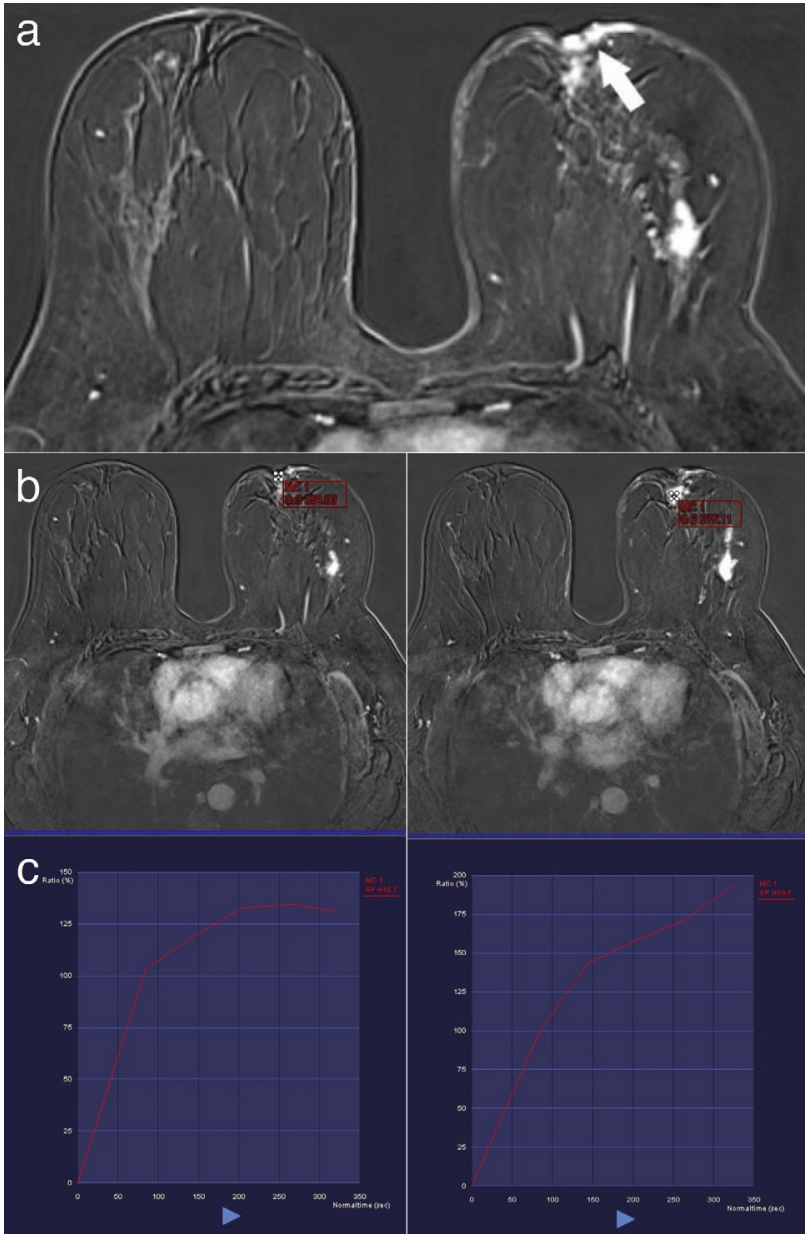

Figure 3. MRI of a 50-year-old woman with invasive breast carcinoma and malignant invasion of the nipple-areola complex. (a) Axial dynamic postcontrast T1-weighted subtraction MRI of the left breast shows pathologic irregular enhancement (white arrow) in the nipple-areola complex. Conventional kinetic analysis by time-intensity curve shows (b) plateau pattern of pathologic NAC enhancement and (c) persistent enhancement pattern for the closest concomitant mass lesion

MRI: Magnetic resonance imaging; NAC: Nipple-areola complex
Continuous enhancement between the NAC to the concomitant malignant lesion was seen in 22 patients: $13(81.3 \%)$ in the PDB group and nine $(81.8 \%)$ in the MTION group $(\mathrm{p}=1.00)$.

The distribution of pathological types of concomitant malignant lesions is shown in Table 1. DCIS was diagnosed in nine $(56.3 \%)$ patients with PDB and one (9.1\%) of the patients with MTION. Invasive ductal carcinoma (IDC) was detected in three patients with PDB (18.8\%) and five patients with MTION (45.5\%). The distribution of pathological types of concomitant malignancy differed significantly between the groups $(\mathrm{p}=0.025)$.

Preoperative breast MRI examinations were conducted with a $1.5-\mathrm{T}$ MR scanner in 17 patients and a 3.0-T MR scanner in 10 patients. Furthermore, 13 of 17 patients examined with 1.5-T MRI were patients with $\mathrm{PDB}$ (76.4\%), seven of 10 patients examined with 3.0-T MRI were patients with MTION (70\%) ( $\mathrm{p}=0.04)$. Areolarperiareolar skin thickening was detected in four $(23.5 \%)$ and eight (80\%) of the patients imaged with $1.5-\mathrm{T}$ and 3.0-T MRI, respectively $(\mathrm{p}=0.007)$. No pathological NAC enhancement was detected in five patients (29.4\%) consisting of four patients with PDB and one patient with MTION imaged with $1.5 \mathrm{~T}$ MRI and three patients with MTION (30\%) imaged with 3.0 T MRI ( $\mathrm{p}=1.00$ ) (Table 3). There were no statistically significant differences in nipple change; the classification, thickness, and kinetic pattern of NAC enhancement; the morphological pattern, size, enhancement kinetic pattern, and pathological diagnosis of concomitant malignant lesion; the similarity of enhancement kinetics pattern of the NAC and concomitant malignant lesions; nipple-to-malignant lesion distance; and the relationship between enhancement of the NAC and concomitant malignant lesions between the patients imaged with 1.5-T and 3.0-T MR (Table 4).

\section{Discussion and Conclusion}

Nipple inversion and retraction are terms used to describe changes in the nipple. Retraction is defined as partial pulling in of the nipple, whereas inversion is complete pulling in. Nipple inversion and retraction may occur secondary to malignant lesions, as well

Table 2. Comparison of concomitant malignant lesions and nipple-areola complex MRI findings according to pathologic diagnosis of nipple-areola complex involvement

\begin{tabular}{|c|c|c|c|}
\hline \multirow[b]{2}{*}{ MRI findings ( $\mathrm{n}$ ) } & \multicolumn{3}{|c|}{ Pathologic diagnosis of NAC involvement } \\
\hline & $\begin{array}{c}\text { PDB } \\
(n=16)(\%)\end{array}$ & $\begin{array}{c}\text { MTION } \\
(n=11)(\%)\end{array}$ & p-value \\
\hline Nipple-to-malignant lesion distance $(\mathrm{cm})$, mean \pm SD & $2.88 \pm 6.40$ & $0.69 \pm 1.63$ & 0.799 \\
\hline \multicolumn{4}{|c|}{ Relationship between enhancement of the NAC and concomitant malignant lesions } \\
\hline Continuous $(n=22)$ & $13(81.3 \%)$ & $9(81.8 \%)$ & \multirow{2}{*}{1.000} \\
\hline Discontinuous $(n=5)$ & $3(18.8 \%)$ & $2(18.2 \%)$ & \\
\hline \multicolumn{4}{|c|}{ Enhancement kinetic pattern of the NAC and concomitant malignant lesions } \\
\hline Same $(n=10)$ & $7(43.8 \%)$ & $3(27.3 \%)$ & \multirow{2}{*}{0.448} \\
\hline Different $(n=17)$ & $9(56.3 \%)$ & $8(72.7 \%)$ & \\
\hline
\end{tabular}

NAC: Nipple-areola complex; MRI: Magnetic resonance imaging; PDB: Paget's disease of the breast; MTION: Malignant tumor invasion of the nipple-areola complex; SD: Standard deviation; n: Number 
Table 3. Comparison of presence of pathological nipple-areola complex enhancement according to magnetic field strength of MRI scanner

\begin{tabular}{|c|c|c|c|c|c|}
\hline \multirow[b]{2}{*}{ Pathological NAC enhancement (n) } & \multicolumn{2}{|c|}{$1.5-T(n=17)$} & \multicolumn{2}{|c|}{$3.0-T(n=10)$} & \multirow[b]{2}{*}{ p-value } \\
\hline & $\begin{array}{c}\text { PDB } \\
(n=13)(\%)\end{array}$ & $\begin{array}{c}\text { MTION } \\
(n=4)(\%)\end{array}$ & $\begin{array}{c}\text { PDB } \\
(n=3)(\%)\end{array}$ & $\begin{array}{c}\text { MTION } \\
(n=7)(\%)\end{array}$ & \\
\hline Absent & $4(23.5 \%)$ & $1(5.8 \%)$ & $0(0.0 \%)$ & $3(30 \%)$ & \multirow{2}{*}{1.000} \\
\hline Present & $9(52.9 \%)$ & $3(17.6 \%)$ & $3(30 \%)$ & $4(40 \%)$ & \\
\hline
\end{tabular}

NAC: Nipple-areola complex; PDB: Paget's disease of the breast; MTION: Malignant tumor invasion of the nipple-areola complex; MRI: Magnetic resonance imaging; n: Number

Table 4. MRI findings compared by magnetic field strength of MRI scanner

\begin{tabular}{|c|c|c|c|}
\hline \multirow[b]{2}{*}{ MRI findings (n) } & \multicolumn{3}{|c|}{ Magnetic field strength of MRI scanner } \\
\hline & $1.5-T(n=17)(\%)$ & $3.0-T(n=10)(\%)$ & p-value \\
\hline \multicolumn{4}{|l|}{ Nipple change } \\
\hline None $(n=8)$ & $6(35.3 \%)$ & $2(20 \%)$ & \multirow{3}{*}{0.877} \\
\hline Inversion ( $n=11)$ & $6(35.3 \%)$ & $5(50 \%)$ & \\
\hline Retraction $(n=8)$ & $5(29.4 \%)$ & $3(30 \%)$ & \\
\hline \multicolumn{4}{|c|}{ Areolar/periareolar skin thickening } \\
\hline Absent $(n=15)$ & $13(76.5 \%)$ & $2(20 \%)$ & \multirow{2}{*}{0.007} \\
\hline Present $(n=12)$ & $4(23.5 \%)$ & $8(80 \%)$ & \\
\hline \multicolumn{4}{|l|}{ NAC enhancement } \\
\hline \multicolumn{4}{|l|}{ Classification } \\
\hline None $(n=1)$ & $1(5.9 \%)$ & $0(0 \%)$ & \multirow{6}{*}{0.299} \\
\hline Normal $(n=7)$ & $4(23.5 \%)$ & $3(30 \%)$ & \\
\hline Nodular $(n=4)$ & $4(23.5 \%)$ & $0(0 \%)$ & \\
\hline Discoid $(n=3)$ & $2(11.8 \%)$ & $1(10 \%)$ & \\
\hline Linear $(n=2)$ & $2(11.8 \%)$ & $0(0 \%)$ & \\
\hline Irregular $(n=10)$ & $4(23.5 \%)$ & $6(60 \%)$ & \\
\hline Thickness (mm), mean \pm SD & $3.80 \pm 2.52$ & $4.61 \pm 2.67$ & 0.438 \\
\hline \multicolumn{4}{|l|}{ Kinetic pattern } \\
\hline Persistent $(n=13)$ & $7(43.7 \%)$ & $6(60 \%)$ & \multirow{3}{*}{0.770} \\
\hline Plateau $(n=8)$ & $6(37.5 \%)$ & $2(20 \%)$ & \\
\hline Washout $(n=5)$ & $3(18.8 \%)$ & $2(20 \%)$ & \\
\hline \multicolumn{4}{|l|}{ Concomitant malignant lesion } \\
\hline \multicolumn{4}{|l|}{ Morphological pattern } \\
\hline Single mass $(n=3)$ & $1(5.9 \%)$ & $2(20 \%)$ & \\
\hline NME $(n=17)$ & $12(70.6 \%)$ & $5(50 \%)$ & \multirow{2}{*}{0.528} \\
\hline NME + mass $(n=7)$ & $4(23.5 \%)$ & $3(30 \%)$ & \\
\hline Maximum size $(\mathrm{mm})$, mean $\pm S D$ & $26.3 \pm 14.4$ & $35.0 \pm 24.8$ & 0.258 \\
\hline \multicolumn{4}{|l|}{ Enhancement kinetic pattern } \\
\hline Persistent $(n=7)$ & $5(29.4 \%)$ & $2(30 \%)$ & \multirow{3}{*}{0.211} \\
\hline Plateau $(n=10)$ & $8(47.1 \%)$ & $2(20 \%)$ & \\
\hline Washout $(n=10)$ & $4(23.5 \%)$ & $6(60 \%)$ & \\
\hline
\end{tabular}


Table 4. Continued

Magnetic field strength of MRI scanner

MRI findings ( $n$ )

\section{Pathological diagnosis}

Inflammatory carcinoma $(n=2)$

Invasive ductal carcinoma $(n=8)$

DCIS $(n=10)$

Invasive breast carcinoma $(n=6)$

Invasive lobular carcinoma ( $\mathrm{n}=1)$

Nipple-to-malignant lesion distance $(\mathrm{cm})$, mean \pm SD
$1.5-\mathrm{T}(\mathrm{n}=17)(\%)$

$2(11.8 \%)$

3 (17.6\%)

8 (47.1\%)

3 (17.6\%)

$1(5.9 \%)$

$2.85 \pm 6.19$
$3.0-\mathrm{T}(\mathrm{n}=10)(\%)$

p-value

$0(0 \%)$

$5(50 \%)$

2 (20\%)

0.258

$3(30 \%)$

0 (0\%)

$0.50 \pm 1.58$

0.354

13 (76.5\%)

9 (90\%)

$1(10 \%)$

0.621

4 (23.5\%)

$3(30 \%)$

7 (70\%)

0.692

$10(58.8 \%)$

$3(30 \%)$

$7(70 \%)$

MTION ( $n=11)$

0.040

NAC: Nipple-areola complex; MRI: Magnetic resonance imaging; PDB: Paget's disease of the breast; MTION: Malignant tumor invasion of the nipple-areola complex; SD: Standard deviation; NME: Nonmass enhancement; DCIS, Ductal carcinoma in situ; n: Number

as congenital or benign causes, and a detailed history and physical examination are crucial to distinguish them (3). In the literature, central, symmetric, slit-like, long-term retraction is generally associated with benign processes, whereas rapid inversion with distortion of the areola usually indicates malignancy $(1,3)$. Although the difference was not statistically significant, nipple inversion on MRI was more frequent among patients with pathologically proven MTION than those with pathologically proven PDB in our study. In a study by Moon et al. (12), nipple change was detected in all six patients with pathologically proven MTION and six of 10 patients with pathologically proven PDB. Contrary to our study, they did not subdivide nipple changes as inversion and retraction. However, the rates of nipple changes in the two patient groups were similar to those in our study.

Areolar-periareolar skin thickening on MRI was significantly detected more frequently in patients with MTION than in patients with PDB. These findings are consistent with those of Moon et al. (12), although the difference between the two groups in their study was smaller compared to that in our study. Patients with MTION may exhibit more edema and areolar-periareolar skin thickening because MTION involves all layers of the skin or areolar lymphatics, whereas PDB is located within the epidermis of the NAC $(2,14,15)$.

Asymmetric NAC enhancement has been emphasized in the literature as a significant indicator of NAC invasion $(5,7,16)$. Symmetric NAC enhancement, which is accepted as normal, was more common in patients with PDB and MTION in our study compared to that in the literature. This may be related to the stage or extent of the disease in the patients analyzed. However, these were not investigated in our study. We compared the presence and types of NAC enhancement in pathologically proven $\mathrm{PDB}$ and MTION cases. Irregular NAC enhancement was more common than the other patterns in both groups, and no significant difference in distribution was found between the groups.

Due to the small size of the NAC area and motion artifacts, kinetic curve assessment may not be reliable for NAC enhancement. Therefore, NAC enhancement kinetics have not been adequately studied and we were not able to find adequate literature data to compare with our findings. In our study, we observed a significant difference between the groups, with persistent enhancement being more common in MTION and plateau pattern more common in PDB. Contrary to our results, Echevarria et al. (17) detected persistent nipple enhancement in two of three patients with PDB, whereas plateau enhancement was detected in only one patient with PDB.

In terms of concomitant malignancies, DCIS was more frequently detected in patients with PDB than those with MTION in our study. DCIS was reported as the concomitant malignancy in $50 \%$ of PDB cases in the study by Moon et al. (12), in two of three patients with PDB in the study by Echevarria et al. (17), and in $88 \%$ of PDB cases in a study by Frei et al. $(18)(2,17)$. These results are compatible with our study.

DCIS usually appears as NME on breast MRI (19, 20). High prevalence of DCIS in patients with PDB was observed in our study; further, the prevalence of NME was higher in the PDB group than in the MTION group. Moon et al. (12) reported that multiple masses were most common in PDB cases. This difference may explain the lower prevalence of DCIS among patients with pathologically proven PDB in their study than in ours. 
In MTION, the malignant tumor either invades the NAC directly or through ducts or lymphatics (6). In contrast, MRI findings of NAC involvement in PDB are mostly associated with eczematous and inflammatory changes of the nipple (13). Therefore, we expected that tumor and NAC contrast enhancement kinetics would be more similar in MTION than PDB; however, we found the opposite. This result may be related to the technically difficult nature of nipple enhancement kinetics analysis due to the small size of the NAC and motion degradation artifacts.

In the literature, it has been reported that a distance of more than $2 \mathrm{~cm}$ between the tumor and NAC and tumor size greater than 2 $\mathrm{cm}$ may increase the risk of malignant NAC invasion $(5,12)$. In our study, we compared these parameters in pathologically proven PDB and pathologically proven MTION cases and found no statistically significant difference. However, we noted smaller distances between the tumor and NAC in MTION cases.

In the present study, enhancement of the NAC and the concomitant malignant lesion was predominantly continuous in both patient groups. In most studies in the literature, continuous enhancement was found to be correlated with NAC invasion $(5,7,16,21)$. However, pathologically proven cases of MTION and PDB were not compared. Moon et al. (12) reported that discontinuous enhancement was more prevalent in pathologically proven MTION, whereas continuous and discontinuous enhancement patterns were equally represented in pathologically proven PDB cases.

3.0-T MRI has higher spatial and temporal resolution than 1.5-T MRI $(22,23)$. The evaluation of the morphology of the breast lesions improved with 3.0-T MRI $(22,23)$. The spectral separation of fat and water is better in 3.0-T MRI. Therefore, fat suppression is superior in 3.0-T MRI images and lesion enhancements are more clearly visualized (23). In our study, the number of patients with increased areolar-periareolar skin thickness was statistically significantly higher in patients imaged with 3.0-T MRI than those imaged with 1.5-T MRI. This may be because of the higher resolution of 3.0-T MRI or that $70 \%$ of patients imaged with 3.0-T MRI were patients with MTION, in whom we found a more frequent increase in areolar-periareolar skin thickness. To clarify this, patients should have been examined with both 1.5-T and 3.0-T MRI and the obtained findings compared. This was not possible because our study was retrospective. In our study, no significant difference was found between 1.5-T and 3.0-T MRI in detecting pathological NAC enhancement in nipple malignancy. When we examined the pathology reports, we found that all cases in which pathological findings in the NAC could not be detected with 1.5-T and 3.0-T MRI were in the early phase of the disease.

Our study had several limitations. First, the sample was selected retrospectively from patients with pathologically proven MTION and PDB who underwent preoperative breast MRI. Although the radiologists were blinded to patient groups while reevaluating the breast MRI images, they knew that all patients had a diagnosis of NAC malignancy. This may have caused bias in the evaluation. Second, since our study was retrospective, we could not evaluate the possible contribution of the results to treatment management and planning for these patient groups. Third, the study population was small. Since $\mathrm{PDB}$ is a rare NAC disease and preoperative MRI of every patient in both patient groups is required, the study population was small. A prospective study with a larger sample size is warranted to overcome
In conclusion, areolar-periareolar skin thickening and asymmetric NAC enhancement with persistent kinetics curve were significant MRI findings for MTION in patients diagnosed with IDC. In contrast, the plateau kinetic pattern of asymmetric NAC enhancement without areolar-periareolar skin changes may support PDB in patients diagnosed with DCIS. 3.0-T MRI was superior to $1.5-\mathrm{T}$ MRI in detecting the presence of areolar-periareolar skin thickness.

Ethics Committee Approval: Approval was received for this study from the Medical Specialty Education Board of Dr. Abdurrahman Yurtaslan Ankara Oncology Training and Research Hospital (2020-10/834).

Informed Consent: Retrospective study.

Peer-review: Externally peer-reviewed.

\section{Authorship Contributions}

Conception: A.C.B.; Design: A.C.B., H.A.; Supervision: A.C.B., H.A.; Materials: I.E.B.; Data Collection and/or Processing: A.C.B., I.E.B., Ö.T., D.S.Ö.; Analysis and/or Interpretation: A.C.B.; Literature Review: A.C.B.; Writing: A.C.B.; Critical Review: H.A.

Conflict of Interest: The authors declare that they have no conflict of interest.

Financial Disclosure: The authors declared that this study received no financial support.

\section{References}

1. Da Costa D, Taddese A, Cure ML, Gerson D, Poppiti R, Esserman LE. Common and unusual diseases of the nipple-areolar complex. Radiographics 2007; 27(Suppl 1): S65-S77. doi: 10.1148/rg.27si075512 (PMID: 18080236) [Crossref]

2. Stone K, Wheeler A. A review of anatomy, physiology, and benign pathology of the nipple. Ann Surg Oncol 2015; 22: 3236-3240. (PMID: 26242366) [Crossref]

3. Nicholson BT, Harvey JA, Cohen MA. Nipple-areolar complex: normal anatomy and benign and malignant processes. Radiographics 2009; 29: 509-523. (PMID: 19325062) [Crossref]

4. Friedman EP, Hall-Craggs MA, Mumtaz H, Schneidau A. Breast MR and the appearance of the normal and abnormal nipple. Clin Radiol 1997; 52 : 854-861. (PMID: 9392464) [Crossref]

5. Cho J, Chung J, Cha ES, Lee JE, Kim JH. Can preoperative 3-T MRI predict nipple-areolar complex involvement in patients with breast cancer? Clin Imaging 2016; 40: 119-124. (PMID: 26423158) [Crossref]

6. Santini D, Taffurelli M, Gelli MC, Grassigli A, Giosa F, Marrano D, et al. Neoplastic involvement of nipple-areolar complex in invasive breast cancer. Am J Surg 1989; 158: 399-403. (PMID: 2817219) [Crossref]

7. Machida Y, Shimauchi A, Igarashi T, Hoshi K, Fukuma E. Preoperative breast MRI: reproducibility and significance of findings relevant to nipple-areolar complex involvement. Breast Cancer 2018; 25: 456-463. (PMID: 29464515) [Crossref]

8. Seetharam S, Fentiman IS. Paget's disease of the nipple. Womens Health (Lond) 2009; 5: 397-402. (PMID: 19586431) [Crossref]

9. Challa VR, Deshmane V. Challenges in diagnosis and management of Paget's disease of the breast: A retrospective study. Indian J Surg 2015; 77 : 1083-1087. (PMID: 27011515) [Crossref]

10. Morandi L, Pession A, Marucci GL, Foschini MP, Pruneri G, Viale G, et al. Intraepidermal cells of Paget's carcinoma of the breast can be genetically different from those of the underlying carcinoma. Hum Pathol 2003; 34: 1321-1330. (PMID: 14691919) [Crossref] 


\section{Coşkun Bilge et al. MRI Findings of Nipple Malignancies}

11. Lim HS, Jeong SJ, Lee JS, Park MH, Kim JW, Shin SS, et al. Paget disease of the breast: mammographic, US, and MR imaging findings with pathologic correlation. Radiographics 2011; 31: 1973-1987. (PMID: 22084182) [Crossref]

12. Moon JY, Chang YW, Lee EH, Seo DY. Malignant invasion of the nipple-areolar complex of the breast: usefulness of breast MRI. AJR Am J Roentgenol 2013; 201: 448-455. (PMID: 23883228) [Crossref]

13. Gao Y, Brachtel EF, Hernandez O, Heller SL. An analysis of nipple enhancement at breast MRI with radiologic-pathologic correlation. Radiographics 2019; 39: 1-18. (PMID: 30547729) [Crossref]

14. Rakha EA, Miligy IM, Gorringe KL, Toss MS, Green AR, Fox SB, et al. Invasion in breast lesions: the role of the epithelial-stroma barrier. Histopathology 2017; 72: 1075-1083. (PMID: 29177112) [Crossref]

15. Sanders MA, Brock JE, Harrison BT, Wieczorek TJ, Hong X, Guidi AJ, et al. Nipple-invasive primary carcinomas: Clinical, imaging, and pathologic features of breast carcinomas originating in the nipple. Arch Pathol Lab Med 2018; 142: 598-605. (PMID: 29431468) [Crossref]

16. Lim S, Park G, Choi HJ, WJ Kwon, Kang BS, Bang M. Use of preoperative mammography, ultrasonography, and MRI to predict nipple areolar complex involvement in breast cancer. Br J Radiol 2019; 92: 20190074. doi: 10.1259/bjr.20190074 (PMID: 31317763) [Crossref]

17. Echevarria JJ, Lopez-Ruiz JA, Martin D, Imaz I, Martin M. Usefulness of MRI in detecting occult breast cancer associated with Paget's disease of the nipple-areolar complex. Br J Radiol 2004; 77: 1036-1039. (PMID: 15569646) [Crossref]

18. Frei KA, Bonel HM, Pelte MF, Hylton NM, Kinkel K. Paget disease of the breast: findings at magnetic resonance imaging and histopathologic correlation. Invest Radiol 2005; 40: 363-367. (PMID: 15905723) [Crossref]

19. Amornsiripanitch N, Lam DL, Rahbar H. Advances in breast MRI in the setting of ductal carcinoma in situ. Semin Roentgenol 2018; 53: 261-269. (PMID: 30449344) [Crossref]

20. Rahbar H, Parsian S, Lam DL, Dontchos BN, Andeen NK, Rendi MH, et al. Can MRI biomarkers at 3 Tesla identify low risk ductal carcinoma in situ? Clin Imaging 2016; 40: 125-129. (PMID: 26365872) [Crossref]

21. Sakamoto N, Tozaki M, Hoshi K, Fukuma E. Is MRI useful for the prediction of nipple involvement? Breast Cancer 2013; 20: 316-322. (PMID: 22311582) [Crossref]

22. Pinker K, Grabner G, Bogner W, Gruber S, Szomolanyi P, Trattnig S, et al. A combined high temporal and high spatial resolution 3 Tesla MR imaging protocol for the assessment of breast lesions: initial results. Invest Radiol 2009; 44: 553-558. (PMID: 19652611) [Crossref]

23. Butler RS, Chen C, Vashi R, Hooley RJ, Philpotts LE. 3.0 Tesla vs 1.5 Tesla breast magnetic resonance imaging in newly diagnosed breast cancer patients. World J Radiol 2013; 5: 285-294. (PMID: 24003354) [Crossref] 